\title{
Modeling and Management of Production Networks
}

\author{
E. Ilie-Zudor, L. Monostori \\ Computer and Automation Research Institute, Hungarian Academy of Sciences, Budapest, \\ Hungary \\ Tel: +361-4665644,Fax: +361-4667503, e-mail: \{laszlo.monostori,ilie\}@sztaki.hu
}

Abstract: The paper attempts to illustrate the concept behind a newly emerging area of research, the concept of production networks. The materialization of this paradigm requires clear definitions of the terms involved, setting up a reference architecture, design and development of supporting platforms and appropriate control methodologies with protocols and mechanisms. The main goal of the paper is to overview the different kinds of production networks, with special emphasis on architectures, advantages and open problems.

\section{INTRODUCTION}

One of the newly emerging areas of research, representing a real challenge for the planning and management of production systems, is related to the paradigm of production networks. Regarding the concept of production networks, different, interrelated concepts can be found in the literature: i.e., virtual enterprise, supply chain, extended enterprise. For the time being, there is not a unique, universally accepted definition yet for these terms. Usually, no clear distinction is made between virtual and extended enterprises, though everyone distinguishes the concepts of virtual factories (enterprises) and virtual manufacturing.

Extended enterprises, virtual enterprises and supply chains are considered in the paper as very similar concepts, each of them being a production network $(P N)$ formed from independent companies collaborating by sharing information, skills, resources, and having the same goal of exploiting market opportunities. A kind of production network focusing on collaboration between companies, is a virtual enterprise (VE). If the collaboration network incorporates a dominant company imposing the rules of the information exchange, we call it extended enterprise (EE). The alliance that focuses on the chain aspects is then the supply chain (SC).

\section{DEFINITIONS AND CATEGORIZATION 2.1 Definitions}

In the related literature, a quite large number of definitions is presented. In the following we specify some of them.

Virtual enterprise

In [3] a virtual factory is defined as a community of dozens of factories each focusing on what it does best, all linked by an electronic network that 
would enable them to operate as one, flexibly and inexpensively regardless of their location.

A virtual enterprise is a temporary consortium formed by real autonomous companies on the basis of strong collaboration to exploit fast changing worldwide opportunities quickly, which a single company is unlikely to realize [35]. Other definitions of the term "virtual enterprise" can be also found in [33], [17], [34], [18], [4], [13].

\section{Supply chain}

The supply chain is a set of activities, which span enterprise functions from the ordering and receipt of raw materials through the manufacturing of products through the distribution and delivery to the customer [10].

The supply chain of a manufacturing enterprise can be defined as a world-wide network of suppliers, factories, warehouses, distribution centers and retailers through which raw materials are acquired, transformed into products which are then delivered to customers [28].

Other definitions of the term "supply chain" can be also found in [2] and [5].

\section{Extended enterprise}

In [32] the virtual enterprise and the extended enterprise are considered as two different notions. The extended enterprise is seen as a network of closely collaborating independent partners, where the goal is to achieve competitive advantages by forming formal linkages (contracts) and to maintain distributed cooperation throughout the network. An enterprise usually belongs to more than one network.

\section{Virtual enterprise- Supply chain}

According to [28] the Supply Chain Management focuses on the chain aspects and is related to the life cycle of products, while the Virtual Enterprise focuses on the collaboration among the related manufacturing factories.

\section{Virtual enterprise - Extended enterprise}

The difference between the extended enterprise and the virtual enterprise is that the virtual enterprise lasts shorter period of time, and has less formal partnerships [32].

The concept of extended enterprise, the closest "rival" term, is better applied to an organization in which a dominant enterprise "extends" its boundaries to all or some of its suppliers. The VE can be seen as a more general concept including other types of organizations, namely a more democratic structure where the cooperation is peer to peer [7].

\section{Virtual enterprise- Virtual manufacturing}

A virtual manufacturing system is defined as a computer system, which can generate the same information about manufacturing system's structure, states, and behaviors as we can observe in a real manufacturing system [13]. The virtual manufacturing model can be used for creating a test field for conducting experiments on the extended enterprise models. 


\subsection{Classification}

The different types of alliances between enterprises can be categorized not only as virtual, extended, supply chain, but also according to criteria presented in Figure 1.

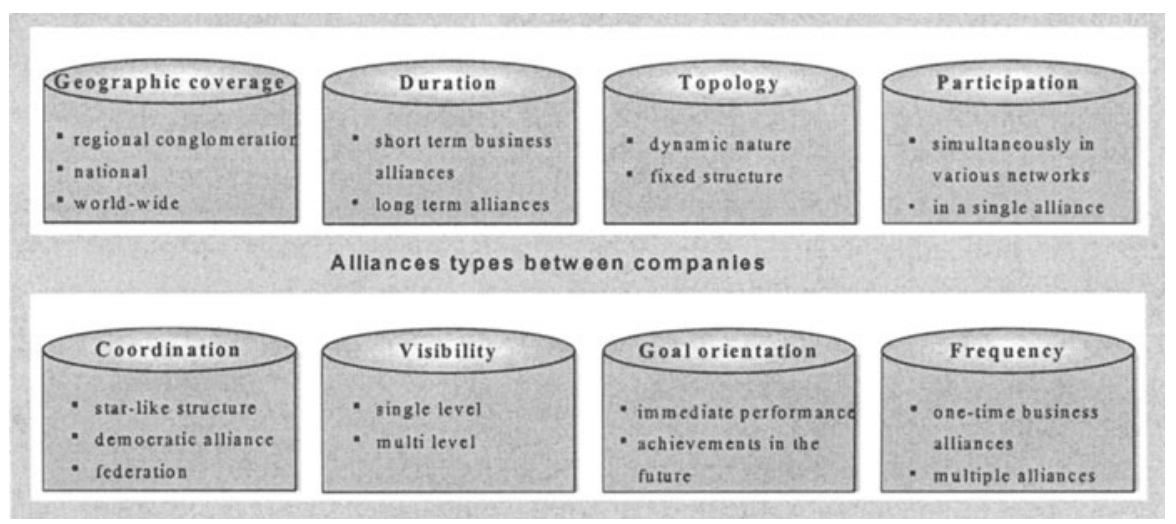

Figure 1. Categorization of alliances according to different criteria

In a dynamic type production network, enterprises can dynamically join or leave the alliance according to the phases of the business process or other market factors.

The structure of the star-like alliance has in the center a dominant company, defining "the rules of the game" and imposing its own standards to the other partners that form a relatively fixed network of suppliers. In a democratic alliance, all participants cooperate on an equal basis, keeping their autonomy, but joining their core competencies. The federation is a common coordination structure created between companies that previously have been partners in a successful alliance and have realized its benefits.

A participant in a single level alliance can only see its direct neighbors, while in a multi level one, has some visibility over other non-direct levels.

\section{APPROACHES, ARCHITECTURES}

The latest approaches to realize extended enterprises, which involve building virtual enterprise architectures, enterprise integration and supply chain management, are based on the agent theory [7],[8],[12],[15], the mobile agents technology [5], [19], [17] or the neural networks technology [13].

\subsection{Virtual enterprise}

\section{Main functional requirements}

In [8] and [7] the main functional requirements for the design of an architecture for industrial virtual enterprises are presented. The work is partly done in the framework of the Prodnet II (Production Planning and Management in an Extended Enterprise, (http://www.uninova.pt/ prodnet/) project. The aim is the development of reference architecture and supporting 
infrastructure for virtual enterprises particularly suited for small and medium sized enterprises in the area of metalomechanics.

The fundamental features of this architecture are incorporated in two main modules: Internal module and Cooperation module. The enterprises in the network are viewed as nodes that add some value to the process. The nodes can exchange information at the same time. Every node is extended with a cooperation module. The Cooperation module supports the information exchange among the nodes. The Internal module is connected to the Cooperation module via a "mapping interface" and the Cooperation modules of different enterprises are connected to the network via a "network infrastructure interface". The Internal Module comprises the complete structure of the company's information and all the internal decision making processes.

Within this architecture two kinds of nodes are defined: Network Coordinator node, as the regulatory component of the enterprise network, and Member Enterprise node, which will store the information about the enterprise itself, and provide the external connectivity.

To support several important information management requirements of the virtual enterprises the PEER [1] federated object-oriented information management system was used.

Agile scheduling

In [17] and [18] a prototype of a multi-agent system for agile scheduling and the extension for the operation in a virtual enterprise environment is described. The system is called MASSYVE (Multi-agent Agile manufacturing Scheduling Systems for Virtual Enterprises) and is part of the MASSYVE INCO-DC KIT Project. MASSYVE uses the HOLOS framework [20] as a baseline for advanced scheduling and the PEER information management framework for the information integration.

The HOLOS [22] scheduling system allows a system to be customtailored for each particular enterprise and, at the same time, to be reconfigured and adapted whenever new production methods, algorithms, production resources, etc., are introduced or changed.

In this framework an instance of a scheduling system is interactively and semi-automatically derived from the HOLOS Generic Architecture (HOLOSGA) [21] reference model, supported by the HOLOS Methodology [22], based on Agent Oriented Programming [30] constructs.

The agent classes used in HOLOS are: the Scheduling Supervisor (SS) (agent that performs the global scheduling supervision and it is the unique system's "door" to other systems), the $\square$ Enterprise Activity Agents (EAA) (the real executors of manufacturing orders), the $\square$ Local Distribution Centers (LDC) (represent functional clusters of EAAs in order to avoid announcement broadcasting, also responsible to select the most suitable agent 
for a certain order after a negotiation process), and the $\square$ Consortium (C) (temporary instances created to supervise the execution of a given order).

HOLOS uses the Contract-Net Protocol coordination mechanism to support the task assignments to agents, and the Negotiation [9], [24] method to overcome conflicts taking place during the scheduling phases. The interaction between the agents is only vertical and agents cannot change the set of other agents that they can communicate with.

The HOLOS control hierarchy uses one global manager (the agent SS), some functional managers (the agents LDC), and assuming that a shop floor is usually composed of production resources (the agents EAA) with small production capacities. A HOLOS agent can establish communications with four kinds of external entities: other HOLOS agents, the end-user, a CIM Information System and the production resources.

MASSYVE proposes a three-layered federated database architecture to support the sharing and exchange of information both within each multi-site enterprise and among different enterprises uniformly, based on PEER: Intraorganization Federated Layer, Federation of HOLOS systems, Federation of Virtual enterprises.

\section{Holonic based framework}

A framework for virtual enterprises based on holonic principles is presented in [12]. The framework is presented through two views: the global and the local view. From the global point of view, authors consider two kinds of holons: Virtual Enterprise (VE) holon and Member Enterprise (ME) holon.

The VE holon is seen as the global coordinator of the virtual enterprise and it is located at the top level of ME holons. The VE holon is not imposing anything to the other holons, but acts as an assistant to them, and provides goals, constraints, warnings, suggestions, knowledge sharing services, etc. The ME holons, from the global view, are located on the same level.

Seen from the local view, holons are distributed on three levels: on the first level the ME holon can be found, on the second level the planning holon and the scheduling holon, and on the third level the task holons and resource holons.

The paper discusses also the VE control under the holonic framework. This includes planning, scheduling, resource sharing, dealing with organizational changes.

\section{Partner selection}

In [15] the problem of choosing the enterprises forming the Virtual Enterprise in a multi-agent environment is discussed. Besides the enterprises, which are seen as agents, the framework comprises a VE coordinator agent and information servers agents. The goal of enterprises is to form the most favorable group that would satisfy a certain need. The Coordinator Agent decomposes the goal in sub-goals and forms a VE goal hierarchy. This hierarchy can be dynamically changed during the process of partner selection. 
The partner selection is treated as a distributed constraint satisfaction problem.

\section{Subcontracting}

In production networks different kind of subcontracting may take place [16] due to technological reasons, and referred as technology-driven subcontracting, due to insufficient capacity, referred as capacity-driven subcontracting, due to strategic reasons for keeping the cooperation with a certain supplier, subcontracting between factories of the same company, etc.

Subcontracting between suppliers and producers, or between producers and customers is called classic subcontracting.

To achieve a better reliability enterprises in the network should allow and assure some information sharing regarding machine loading, availability, orders progress, planned demands, stocks. In order to help increasing the redundancy in networks, a software tool called FAST/net was developed. The software provides information about the status of orders, resource availability, partners' stocks. FAST identifies bottleneck systems, marks orders suitable for subcontracting, and makes calculations on security stocks.

Reference model

A reference model for virtual enterprises can be found in [4]. Enterprises searching for business opportunities form an aggregation named Virtual Industry Cluster (VIC). When a business opportunity can be exploited, a Virtual Enterprise Broker (VEB) will search through different Virtual Industry Clusters for enterprises with the appropriate competencies. The enterprises in question will form a Virtual Enterprise (VE). When a VIC has a VEB of its own, the VIC becomes a Virtual Organization (VO). The number of enterprises in a VIC is varying, enterprises may enter or exit the aggregation at any time.

The VEB obtains and offers business opportunities to the members of VIC and sets up the VE. The VIC provides information about the competencies of the enterprises involved. The VE will run the business opportunities and take part in its reconfiguration.

The VO has to manage alliances, and define and manage strategies.

\subsection{Internet enabled virtual enterprises and supply chains}

The Collaborative Agent System Architecture (CASA) and the Infrastructure for Collaborative Agent Systems (ICAS) have been developed for implementing Internet enabled virtual enterprises and for managing the Internet enabled complex supply chain for a large manufacturing enterprise, though, initially they were proposed as a general approach for Internet based collaborative agent systems [28], [27].

The approach comprises the following elements: Cooperation Domain Servers, Yellow Page Agents, Local Area Coordinators, Collaborative 
Interface Agents, High-Level Collaboration Agents, Knowledge Management Agents, Ontology Server.

The cooperation domain server receives all the messages sent by the agents in the cooperation domain and forwards the message transparently to its destination and may also record all the transactions.

Yellow Page agents accept messages for registering services and record this information in a local database.

A local area coordinator acts both as a representative of the area to the outside world, and a manager for the local agents within the area. It also provides an interface service to the outside world.

Collaborative Interface Agents $\square$ are supposed to be communicative, semiautonomous, collaborative, reactive, pro-active, adaptive, self-aware and mobile. High-Level Collaboration Agents are introduced to increase the basic collaboration services provided by Yellow Page Agents and Local Area Coordinators agents. Agents do not communicate with each other directly, but those working together form cooperation domains. Each agent in a cooperation domain routes all its outgoing messages through the cooperation domain server. All Cooperation Domain Servers are connected with the Ontology Server for translating messages from different agents into a common format.

Five mechanisms developed in previous projects (MetaMorph, MetaMorph I, MetaMorph II) are used: Agent-Based Mediator-Centric Organization, Task Decomposition, Virtual Clustering, Partial Agent Cloning, Adaptation and Learning.

\subsection{Supply chain}

A hybrid agent-based architecture for manufacturing enterprise integration and supply chain management is proposed in the framework of MetaMorph II (http://imsg.enme.ucalgary.ca/research.htm\#top) project [25], [29]. The objective of the project is to integrate the manufacturing enterprise's activities such as design, planning, scheduling, simulation, execution, and product distribution, with those of its suppliers, customers and partners into an open, distributed intelligent environment. The architecture was named Agent-Based Manufacturing Enterprise Infrastructure (ABMEI).

MetaMorph II architecture considers a main manufacturing enterprise central to the supply chain management. Each manufacturing enterprise has to have at least one Enterprise Mediator. Partners, suppliers and customers are dynamically connected with this main enterprise through other kind of mediators via the Internet/Intranet.

Mediators are agents, called mediator agents. They provide primarily message services and promote cooperation among intelligent agents, furthemore learning from the agents' behavior. All manufacturing resources (e.g.: machines, tools, workers, AGVs, etc.) are modeled as resource agents and are coordinated by layered mediator agents. Parts are also modeled as 
agents. Part agents do not communicate directly with resource agents. All manufacturing task requests are sent to the Resource Mediators.

MetaMorph II combines the mediation mechanism based on hierarchical mediators and the bidding mechanism based on the Contract Net protocol to solve the cooperative negotiation among resource agents. The authors propose two scheduling mechanisms: Machine-Centered Scheduling and Worker-Centered Scheduling. The present implementation of ABMEI consists of four mediators: Enterprise Mediator, Design Mediator, Resource Mediator and Marketing Mediator.

\section{Static and mobile agents infrastructure}

A supply chain infrastructure in a textile-manufacturing environment is presented in [5]. The framework consists of static and mobile cooperating agents, organized in three functional levels: the supply chain level, the factory level and the user level. Mobile agents have behavior, state and location. Agents share the same communication language and a common vocabulary. They use code mobility as well.

\section{Generic reusable enterprise model}

The architecture of the Integrated Supply Chain Management System presented in [10] is based on a set of cooperating agents. There are two types of agents: functional agents, for planning and controlling activities in the supply chain, and information agents, for information and communication services. The functional agents in this architecture are the Order Acquisition agent, the Scheduling agent, the Resource Management agent, the Dispatching agent, the Transportation Management agent, and the Logistics agent. Supply chain agents exist within an Enterprise Information Architecture (EIA), having a generic reusable enterprise model in the center, in order to support the integration of supply chain agents.

The EIA is responsible for finding the information that a certain agent asks for, and also for distributing the information an agent wants to share, to the agents that is interested.

\section{ADVANTAGES}

The involvement in production networks like these, has numerous advantages for the member enterprises, such as:

- increased reaction to changes in market condition and demands, because the participation in the network involves a better flexibility and agility;

- possibility to complement core competencies in order to be able to share some market opportunities;

- ability to specialize in few areas, representing the core competencies;

- new market opportunities, by increasing the geographic coverage;

- improved quality and responsiveness to market opportunities;

- elimination of capacity bottlenecks and reduction of capital investment through resource sharing; 
- making global life-cycle orientation possible;

- increased reuse and recycling in waste management;

- increased redundancy, as more than one partner may produce the same service or product.

\section{PROBLEMS, OPEN QUESTIONS}

In every production network, one of the main problems is represented by the information interchange through the network. In addition to this, problems caused by differences in culture, trust issues are added. A scheme of the issues to be considered is presented in Figure 2.
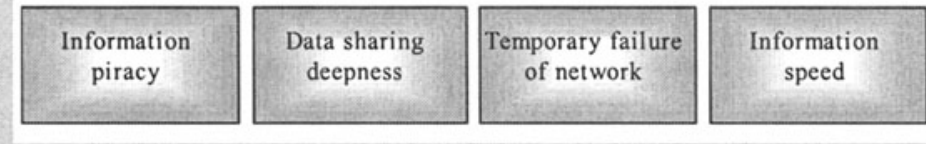

Information

overload

Main problems in production networks
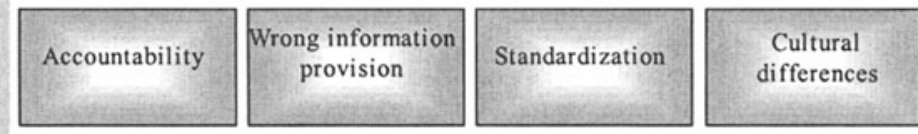

Cultural assumptions

Figure2. Issues to be considered when independent companies join into alliances

\subsection{Information interchange}

Piracy. The security mechanisms and the access rights have to be very well implemented, in order to protect the network against unauthorized access to information, which could lead to secret information reveling, unauthorized utilization of resources, result in the alteration of information without any gain to the intruder.

Deepness. In some cases, partners agree to share data by making internal information visible for each others, such as the capacity of a particular shop floor, the orders progress, material flow status, etc., in order to adjust their own planning. An issue to be dealt with in this case is how deep a partner can allow to the others to see into his internal structure.

Temporary failures. The redundancy of information should be ensured, because temporary failures might happen in the network.

Speed. In order to work as a single integrated unit companies must be able to inter-operate, share and exchange information in real time. As the communication between enterprises may induce high network traffic, it is necessary to speed up the exchange of information.

Overloads. The high network traffic mentioned above can cause not only slowness in the network, but also overloads. Therefore, the data volume in a network has to be limited. This can be done through the reduction of the need for communication or the reduction of details. 
Accountability. It is necessary to guarantee that the sender and/or receiver should not deny the transaction after it has taken place [17].

Incomplete or wrong information provision. Enterprises in the network do not consider the global optimization as their main purpose, but they act selfishly, often having antagonic goals, and therefore, sending wrong or incomplete information on purpose, even though a general willingness to cooperate can be assumed.

Standardization. Because of a different technical background, at the time they enter a production network, companies may use different software, different standards, different communication protocols. In order to exchange and share information with each others, enterprises must agree on the use of some common standards.

At the moment, most enterprise networking experiences are based on the implantation of the STEP standard for the exchange of technical product data and of the EDIFACT standard for commercial data exchange. In agent based system developments, standards as CORBA (Common Object Request Broker Architecture) (http://www.omg.org/corba/) have been used for interagent communication, and for developing agent based manufacturing systems, KQML (Knowledge Query and Manipulation Language, $\mathrm{http} / / / \mathrm{www} . c s . u m b c . e d u / \mathrm{kqml} /$ ) as a common communication language for agents, with KIF as a common content format. The situation regarding quality management has also to be considered.

\subsection{Cultural differences}

Enterprises can enter a PN such as a virtual enterprise e.g. from different geographic areas. In this case, new issues that have to be dealt with will appear: Different legislation in different countries; Different time zones; Different currencies; Different technical and cultural background; Different educational level; Team-work experience, etc.

\subsection{Cultural assumptions}

Between the members of a PN must exist an ethic code for providing a base for trust and the warranty of cooperation between members. Companies are supposed to manifest solidarity, collective orientation, trust [10].

For enterprises (agents), in order to cooperate, there must be cultural assumptions such as [34]: agents are constraint-based problem solvers, agents have the ability to generate more than one solution, agents have the ability and authority to realize local goals and possibly relax a sub-set of constraints if the global solution is further optimized, etc.

\section{CONCLUSIONS}

Different concepts (virtual enterprise, supply chain, extended enterprise) behind the general term of production networks were discussed in the paper, with the aim of making some order in the closely related approaches. Special emphasis was given on the characteristic features of these architectures with 
their advantages and disadvantages. The elimination of the enumerated open problems requires further research efforts combining the approaches of different disciplines, such as information technology, artificial intelligence and operation research.

A list of "live" links related to the concept of production networks (Virtual/Extended Enterprises, Supply Chains) can be found, among other things, on the following web page: http://www.sztaki.hu/sztaki/ake/ai/ manufacturing/.

\section{ACKNOWLEDGEMENTS}

This work was partially supported by the National Research Foundation, Hungary, Grant Nos. T026486 and T034632.

\section{References}

[1] Afsarmanesh, H.; Wiedijk, M.; Hertzeberger, L.O.; Negreiros Gomes, F.; Provedel, A.; Martins, R.C.; Salles, E.O.T. 1995: A Federated Cooperation Architecture for Expert Systems Involved in Layout Optimization. in Balanced Automation Systems, L.M. Camarinha-Matos and H. Afsarmanesh (Eds.), Chapman \& Hall, Jul 1995.

[2] Barbuceanu, M., Fox, M.S., 1997: Dynamic Team Formation and Management in an Agent Structured Supply Chain.

[3] Bell Canada Company, 1996, Harvard Business Review, July/August 1996.

[4] Bremer, C. F., 2000: From An Opportunity Identification to its Manufacturing: A Reference Model for Virtual Enterprises, Annals of the CIRP, vol. 49/1, 2000, pp. 325-329.

[5] Brugali D., Menga G., Galarraga G., 1998: Inter-Company Supply Chain Integration via Mobile Agents., In The Globalization of Manufacturing in the Digital Communications Era of the 21 st Century: Innovation, Agility, and the Virtual Enterprise. Kluwer Academic Pub. 1998, http://www. polito.it/brugali/.

[6] Camarihna-Matos, L.M., Afsarmanesh, H., 2000: Frameworks for Agile Virtual Enterprise in Manufacturing, IFAC-MIM Symposium on Manufacturing, Modeling, Management and Control, Rio, Patras, Greece, 12-14 July, pp. 84-89.

[7] Camarinha-Matos L.M., Afsarmanesh H., Garita C., Lima C., 1997: Towards an Architecture for Virtual Enterprises, Keynote paper, Proc. 2nd World Congress on Intelligent Manufacturing Processes and Systems, Springer, Budapest, Hungary, June 1997, pp. 531-541.

[8] Camarinha-Matos, L.M., Afsarmanesh H., 1997: Virtual Enterprises: Life Cycle Supporting Tools and Technologies, Handbook of Life Cycle Engineering: Concepts, Tools and Techniques, A. Molina, J. Sanchez, A. Kusiak (Eds.), Chapman and Hall

[9] Davis, R.; Smith, R., 1983: Negotiation as a Metaphor for Distributed Problem Solving, Artificial Intelligence, N 20, pp. 63-109.

[10] Fox M.S., Chionglo J.F., and Barbuceanu M., 1993: The Integrated Supply Chain Management System, Internal Report, Dept. of Industrial Engineering, University of Toronto.The Integrated Supply Chain Management Project, http://www.eil.utoronto.ca/iscm-descr.html.

[11] Hongmei G., Biqing H., Wenhuang L., Shoujo R., Yu L., 2000: The Application of Holonic Manufacturing Paradigm to the Virtual Enterprise Control, Proceedings of $16^{\text {th }}$ World Computer Congress 2000, Information Technology for Business Management, Aug 2000, Beijing, China, pp. 270-276.

[12] Kazuaki Iwata, Masahiko Onosato, Koji Teramoto, Suguru Osaki, 1997: Virtual Manufacturing Systems as Advanced Information Infrastructure for Integrating manufacturing Resources and Activities, Annals of the CIRP Vol. 46/1/1997, pp. 335-338.

[13] Lau H.C.W., Chin K.S., Pun K.F., Ning A., 2000: Decision supporting functionality in a virtual enterprise network, Expert Systems with Applications, 19/ 2000, pp. 261-270.

[14] Lutz, S., Wiendahl, H-P., 2000: Concept for Production-Management in Networks, Proceedings of $16^{\text {th }}$ World Computer Congress 2000, Information Technology for Business Management, Aug 2000, Beijing, China, pp. 424-431.

[15] Macgregor, R. S.; Aresi, A.; Siegert, A., 1996: WWW.Security, How to Build a Secure World Wide Web Connection, IBM, Prentice Hall PTR. 
[16] Mezgár I., Kovács G.L., Paganelli P. 2000: Co-operative Production Planning for Small and Medium-Sized Enterprises, International Journal of Production Economics, No. 64, pp. 37-48.

[17] Monostori, L.; Szelke, E.; Kádár, B.: Intelligent techniques for management of changes and disturbances in manufacturing, Proceedings of the CIRP International Symposium: Advanced Design and Manufacture in the Global Manufacturing Era, August 21-22, 1997, Hong Kong, Vol. 1, pp. 67-75.

[18] NIIIP 1999: Vision of the National Industrial Information Infrastructure Protocols (NIIIP), http://www.niiip.org/vision.html.

[19] Rabelo R., Camarinha-Matos L.M., Afsarmanesh H., 1998: Multiagent perspectives to agile scheduling, Proc. Of BASYS' $98-3^{\text {rd }}$ IEEE/IFIP Int. Conf. On Balanced Automation Systems, Intelligent Systems for Manufacturing (Kluwer Academic), pp. 51-66, ISBN 0-412-84670-5, Prague, Czech Republic, Aug 98.

[20] Rabelo R., Camarinha-Matos L.M., Afsarmanesh H., 1999: Multi-agent-based agile scheduling, Journal of Robotics and Autonomous Systems (Elsevier), Vol. 27, N. 1-2, April 1999, ISSN 09218890 , pp. $15-28$.

[21] Rabelo R., Spinosa M., 1997: Mobile-agent based supervision in supply chain management in the food industry, Proceedings of Agrosoft'97 - Workshop on supply chain management in agribusiness, Belo Horizonte, Brazil, Sept. 97, pp. 451-459.

[22] Rabelo, R.J., 1997: A Framework for the Development of Manufacturing Agile Scheduling Systems - A Multi-agent Approach, Ph.D. Thesis, New University of Lisbon, Portugal.

[23] Rabelo, R.J.; Camarinha-Matos, L. M., 1995: A Holistic Control Architecture Infrastructure for Dynamic Scheduling, in Artificial Intelligence in Reactive Scheduling, Eds. Roger Kerr e Elizabeth Szelke, Chapman \& Hall, pp.78-94.

[24] Rabelo, R.J.; Camarinha-Matos, L. M., 1996a: Deriving Particular Agile Scheduling Systems using the HOLOS Methodology, International Journal in Informatics and Control.

[25] Rabelo, R.J.; Camarinha-Matos, L. M., 1998b: Generic framework for conflict resolution in negotiation-based agile scheduling systems, Proceedings IMS'98 - 5 th IFAC Workshop on Intelligent Manufacturing Systems, Gramado - Brazil, pp.187-192.

[26] Shen W., Norrie D.H., 1998: An Agent-Based Approach for Manufacturing Enterprise Integration and Supply Chain Management, In G. Jacucci, et al (eds.), Globalization of Manufacturing in the Digital Communications Era of the 21 st Century: Innovation, Agility, and the Virtual Enterprise, Kluwer Academic Publishers, 1998, pp. 579-590.

[27] Shen, W., Norrie, D.H., 1999: Implementing Internet Enabled Virtual Enterprises using Collaborative Agents, In Camarinha-Matos, L.M. (ed.), Infrastructures for Virtual Enterprises, Kluwer Academic Publisher, pp. 343-352, (http://imsg.enme.ucalgary.ca/ publicate.htm\#ISG publications in 1999).

[28] Shen, W., Ulieru, M., Norrie, D.H. and Kremer, R., 1999: Implementing the Internet Enabled Supply Chain through a Collaborative Agent System, Proceedings of Agents'99 Workshop on Agent Based Decision-Support for Managing the Internet-Enabled Supply-Chain, Seattle, WA, May $1-5$, pp. 55-62.

[29] Shen, W., Xue, D., Norrie, D.H., 1998a: An Agent-Based Manufacturing Enterprise Infrastructure for Distributed Integrated Intelligent Manufacturing Systems, in Proceedings of PAAM'98, London, UK. (A hybrid agent-based approach for integrating manufacturing enterprise activities with its suppliers, partners and customers within an open and dynamic environment. Description of its functional architecture, main features and a prototype implementation.) (http://imsg.enme.ucalgary.ca/).

[30] Shoham, Y., 1993: Agent-Oriented Programming, Artificial Intelligence, N 60, Elsevier, pp.5192.

[31] Szegheo, O., 1999: Extended Enterprise-the Globeman 21 way, Proceedigs of the Second International Workshop on Intelligent Manufacturing Systems, sept. 1999, Leuven, Belgium, pp. 405-410.

[32] Walton, J.; Whicker, L., 1996: Virtual Enterprise: Myth \& Reality, J. Control, Oct 1996.

[33] Wiendahl H.P., Helms K., Höbig M., 1998: Management of Variable Production NetworksVisions, Management and Tools, Annals of the CIRP, vol. 47/2, 1998, pp. 549-555.

[34] Yu L., Biqing H., Wenhuang L., Wu C., Hongmei G., 2000: Multi-Agent System for Partner Selection of Virtual Enterprise, Proceedings of $16^{\text {th }}$ World Computer Congress 2000, Information Technology for Business Management, Aug 2000, Beijing, China, pp. 287-294.

[35] Zhou, Q., Ristic, M., Besant, C.B., 2000: An Information Management Architecture for Production Planning in a Virtual Enterprise, in The International Journal of Advanced Manufacturing Technology, (2000) 16: pp. 909-916. 